\title{
Strates
}

STRATES Matériaux pour la recherche en sciences sociales

$11 \mid 2004$

Jeune recherche, la vitalité d'un laboratoire

\section{Une expérience paysagère : Béjaiia et ses environs, entre violence et douceur de vivre}

Odile BERKI

\section{(2) OpenEdition \\ 12 Journals}

Édition électronique

URL : http://journals.openedition.org/strates/437

ISSN : $1777-5442$

Éditeur

Laboratoire Ladyss

Édition imprimée

Date de publication : 1 janvier 2004

ISSN : 0768-8067

\section{Référence électronique}

Odile BERKI, « Une expérience paysagère : Béjaïa et ses environs, entre violence et douceur de vivre », Strates [En ligne], 11 | 2004, mis en ligne le 14 janvier 2005, consulté le 07 septembre 2020. URL : http://journals.openedition.org/strates/437

Ce document a été généré automatiquement le 7 septembre 2020

Tous droits réservés 


\title{
Une expérience paysagère : Béjaïa et ses environs, entre violence et douceur de vivre
}

\author{
Odile BERKI
}

À Tassadit, Naïma et Maïween, trois femmes, trois générations... « Nous savons tous que le droit est la plus puissante école de l'imagination. Jamais poète n'a interprété la nature aussi librement qu'un juriste la réalité " Jean Giraudoux, La guerre de Troie n'aura pas lieu « Tout est symbole pour celui qui n'a plus rien à

lire»

Kateb Yacine

Pour...

1 Pour avoir hésité à m'y rendre pendant les vacances d'été, pour avoir été surprise, puis charmée, par la beauté des paysages de Béjaïa et de ses environs, pour m'y être sentie chez moi, sans doute parce que j'y ai retrouvé cette capacité humaine de sourire, d'aimer, même face à la douleur ou l'échec, pour y avoir réappris à dépasser l'amertume générée par la rudesse de la vie et sa violence extraordinaire, presque insupportable, à la fois partout présente et qu'on ne voit nulle part, pour y avoir observé, en jouant de ma spécialisation de géographe, ma vie de parisienne, mon expérience de globe-trotteur de naissance et des écarts de pratiques culturelles, je crois pouvoir dire qu'une expérience paysagère forte, telle que je l'ai ressentie à Béjaïa, a une valeur heuristique dont on ne peut rendre compte par des analyses purement conceptuelles. Il y a dans l'attention portée à ce qui se déroule dans la tête, dans le cœur et ce qui vous atteint du dehors un potentiel resté en jachère qu'il faut écouter, déchiffrer, transcrire, transmettre et qui peut stimuler ma réflexion sur la protection des paysages et des Grands Sites en France. Avec mes yeux de jeune chercheuse, j'ai accompagné mon mari chez lui. J'ai découvert une Algérie tout autre que celle que 
j'avais imaginée ou dont on parle dans les médias. Je suis revenue avec le sentiment que nous avions beaucoup à apprendre sur ce pays.

Découvrir Béjaïa et sa baie

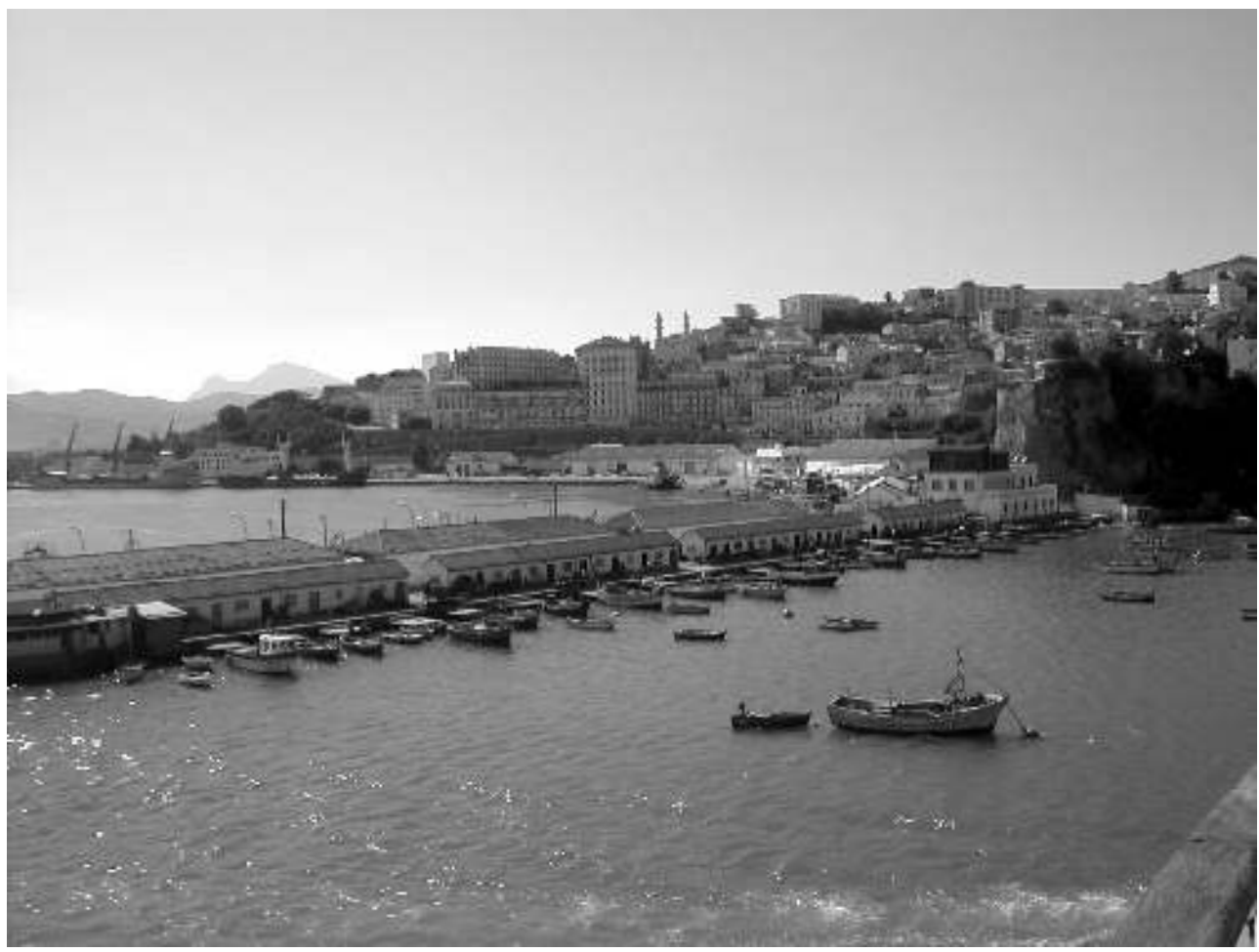

(c) « Une petite ville méditerranéenne bien tranquille ». Photo berki o\&m, 2002.

2 Découvrir Béjaïa et sa baie, depuis l'avion ou le bateau, sont deux expériences paysagères différentes, toutes deux riches en sensations et en émotions.

L'image que j'ai gardée de l'atterrissage à Béjaïa est celle-ci : au bout d'une heure trente de vol on devine le trait de côte algérienne, semblable à d'autres; au fur et à mesure qu'on perd de l'altitude on distingue les plages et la mer bleu turquoise, qu'on imagine aussi chaude et délassante qu'un bain maure, dans laquelle les montagnes de la Petite Kabylie tombent à pic. Au fond, il y a la ville de Béjaïa, « accrochée » à sa presqu'île. Les massifs impressionnants par leur masse, aux belles lignes de crêtes, si présents, donnent à la baie de Béjaïa dont on m'avait dit « c'est une petite ville » une dimension démesurée... Chaque été, avec l'air chaud et humide, j'ai l'impression de sentir le poids de l'Afrique. Atterrir à Béjaïa c'est, pour un instant, être un oiseau qui se pose sur l'épaule d'un géant.

En août 1998, la première fois, j'ai immédiatement pensé au souvenir que je gardais de l'arrivée à Papeete (Tahiti). La ressemblance était troublante : montagnes vertes, mer bleu turquoise, air chaud et humide, aéroport international, chaleur suffocante à l'intérieur du bâtiment climatisé où les cris et les rires se mêlent aux voix des douaniers et aux «bruits » de tampons, de valises ou de sacs traînés à terre. Pas de désert, pas d'oasis... Je découvrais une ville, indissociable de son golfe et de sa position "en bordure d'Afrique", ainsi qu'un pays, celui de mon époux. Ce voyage était bien différent des autres "déracinements" qui m’ont marquée: élevée entre Djibouti, Tunis, Papeete (Tahiti), Port-au-Prince (en Haïti) et Mutsamudu (à Anjouan, aux 
Comores), c'était comme si je retrouvais les lieux de mon enfance, l'art de voyager pour voler de mes propres ailes, comme la fin d'une coupure difficile à vivre.

Le nom de l'aéroport, «Béjaïa - vallée de la Soummam », m’a immédiatement frappée, bien plus que les militaires figés sur le tarmacadam, mitraillettes au corps - j'avais malheureusement pris l'habitude de ces « ambiances » en Haïti sous le régime Duvalier. Ce nom dont j'ignorais tout a été comme le flash du «c'est ça » qui réverbère et se répercute au long d'une représentation de théâtre ${ }^{1}$.

6 Depuis le bateau, le paysage de Béjaïa et ses environs, découvert au « ras du décor ", se compose et se défait à une vitesse infinie. À bord, le "pays-visage " ${ }^{2}$ du bateau change au fur et à mesure que nous nous rapprochons des côtes : il y a nettement moins de femmes sur les ponts, elles ne fument plus.

"Une petite ville tranquille?»

7 «D'après la carte postale que tu m'as envoyée on dirait une petite ville méditerranéenne bien tranquille» m'avait dit Stéphane, alors que j'essayais de répondre à la question attendue et redoutable : «Comment se sont passées tes vacances en Algérie ? »... Béjaïa, « une petite ville tranquille», je n'en croyais pas mes oreilles : entendre cela malgré les images de révoltes qu'on avait pu voir sur les chaînes de télévisions françaises, c'est qu'il y avait une contradiction à dépasser du point de vue du paysage. En effet, ce que j'avais entendu sur Bougie ${ }^{3}$ était une partie de la réalité, une image et une représentation.

8 Beaucoup de faits et de paroles d'habitants portent à croire que la wilaya ${ }^{4}$ de Béjaïa n'est plus considérée comme la « petite Suisse locale ». En 2001, les Algériens (-nes) qui n'y avaient pas d'attache familiale avaient peur de venir. Les stations balnéaires de Tichi ou d'Aokas, habituellement envahies par les touristes, étaient désertes. La bonne réputation s'est envolée avec le "printemps noir " ${ }^{5}$. Évidemment, les avis sont partagés devant la tournure qu'ont pris les événements. Les uns ont été sidérés par la violence qui s'exprimait dans la rue: "Les jeunes déboulonnaient les lampadaires avec leurs mains! C'est dire la colère qu'ils ont accumulée pendant tant d'années » (un ami). Les autres regrettent que les marches pacifiques aient été troublées par les débordements de « petits casseurs ».

9 En ville, les équipements publics forts en symbole ont été incendiés : le centre culturel, l'hôtel des impôts, l'agence Air Algérie, etc. On pouvait encore lire sur les murs ou des banderoles noires: "Ulach smach» [«il n'y a pas de justice »], « 40 ans de mépris... Barakat! » [barakat! : « ça suffit! »], « hogra », c'est-à-dire « honte à ceux qui usent de leur force pour mépriser les autres ». Les gendarmes ont été interdits de séjour par le peuple. Leurs locaux ont été transformés en habitation de fortune. Les HLM non attribués ont été squattés. Les habitants se surveillaient eux-mêmes. C'était comme si la pression militaire était devenue moins visible.

$10 \mathrm{Si}$, pour certains, le mouvement de révolte kabyle a rendu le slogan «Béjaïa, une belle petite ville » obsolète parce qu'il a marqué dans sa matérialité le chef-lieu de wilaya, l'optimisme que renfermait cette expression m'a toujours surprise : le site est en cours de massacre, les héritages architecturaux des différentes colonisations tombent en ruine comme si la patrimonialisation était en dormance. L'espace public non entretenu donne l'impression à un observateur dont l'œil n'est pas habitué qu'il n'y a pas de politique d'urbanisme ; tout porte à croire qu'il n'a jamais été traité ou que le maintien de cet état est volontaire. Les maisons individuelles cubiques poussent comme des champignons. Le «fer à béton dépassant des murs de briques non crépis que l'on 
réhausse parfois d'un étage $»^{6}$ fait partie du paysage urbain : il est en centre ville, dans les quartiers planifiés des années 80 et les quartiers illicites qui vont à l'assaut des collines entourant la vallée de la Soummam. Le mitage dévore l'espace à l'horizontale et à la verticale. «L'architecture est un fait social : chacun construit sa maison, sans tenir compte des règles d'urbanisme. C'est le maçon, le peintre qui s'improvise architecte.» (Malek). Les rues sales, comme si on avait "perdu toute foi en l'efficacité d'un comportement citoyen ${ }^{7}$ sont à certaines heures le lieu de rendez-vous des hommes et des jeunes qui «tiennent les murs du quotidien $»^{8}$. En outre, l'été, il n'y a de l'eau courante qu'aux premières heures du jour, la vie des Algériens étant ponctuée par une épuisante manipulation de seaux alors que chez les grossistes il y a des litres d'eau de sources... Par ailleurs, les eaux usées se mélangent accidentellement et régulièrement à l'eau potable. Rien n'est fait pour l'écoulement des eaux de pluie des orages violents comme ceux du mois de novembre 2001. Un Bougiotte m'a dit : «T'appelles ça un pays toi ? Trois jours sans eau! Dis-moi! Ils nous ont tout volé! Tout !» « Ils » ce sont ceux qui ont intérêt à maintenir la hogra.

11 J'ai souvent été frappée par le contraste entre le gris « ambiant » et la représentation positive que beaucoup de Bougiottes, émigrés ou non, avaient encore de leur ville. Je pensais que Béjaïa avait dû et pourrait être belle mais qu'aujourd'hui il s'agissait d'un vaste chantier.

Violence, paysage et douceur de vivre

12 Je me demande quelle est la signification du paysage dans la société algérienne, qui au regard des événements relatés dans la presse française se structure actuellement dans la violence : comment et dans quelle mesure une société déchirée par les luttes, une " société de la guerre ", qui secrète des moyens de régler les conflits, façonne-t-elle les paysages du territoire où elle évolue? Le désordre visible est-il dans ce pays uniquement l'expression à vif de consciences meurtries qui portent la hantise du sang, débordant l'imaginaire et donnant la pulsation d'un décor jailli de la violence historique? Les conflits font partie de la vie mais le chaos que j'ai vu à Béjaïa, par exemple, peut être conçu dans la vitalité : certains Bougiottes préfèrent le terme " effervescence».

Désordre, effervescence, ordre, paysage, violence muette, "petite Suisse algérienne »... L'image des montagnes kabyles tombant à pic dans la Méditerranée est dans une certaine mesure comparable à celle des Alpes dans le lac Léman. Au-delà de la carte postale, on pourrait établir le parallèle au niveau du sens du paysage : je pense souvent à cette phrase provocatrice et célèbre : « La Suisse est une prison où chaque citoyen est son geôlier »... Si la violence n'est pas la fin ultime du paysage, «sur la rive dorée du lac [de Zurich], dans cette Suisse de l'argent énorme et secret, on peut être 'éduqué à mort'. Pas un mot plus haut que l'autre, pas de rire bruyant et vulgaire, pas de pleurs, pour tout dire déplacés, puisque le monde est, ce monde-là, harmonieux, parfait, comme il faut. Convenances, conventions, contention, tension jusqu'à ce que ça craque $»^{9}$. Je pose la question suivante: des paysages ordonnés produisent-ils une violence de la société ? ${ }^{10}$

14 La violence invisible du paysage, c'est aussi cette dualité qui vit en moi. Lorsque je flâne à Béjaïa, insouciante, à la recherche de " curiosités » architecturales ou que je glane des scènes de vie urbaines, j'éprouve une sensation troublante : je souffre d'être étrangère à la ville, d'être mise au centre de l'attention des Bougiottes un bref instant alors que je cherche le plaisir vertigineux de me fondre dans la foule. Par ailleurs, je sens dans mon 
dos le regard scrutateur d'un observateur impassible. C'est comme si le paysage devenait subjectif et que des rappels à l'ordre muets de sa matérialité en rétablissaient vite l'objectivité. Ce que j'ai vu et vécu en Algérie me permet de dire qu'il s'agit de quelque chose de doux au-delà des images ou des représentations qu'on pourrait avoir de ce pays et auquel on ne peut avoir accès avec ironie.

Pourtant je me demande dans quelle mesure le contraste entre un "pays-visage " urbain masculin et la crête qui surplombe l'ancien centre ville, "Yemma Gouraya " [Yemma: Maman] qui a la forme d'une femme couchée, une «belle au bois dormant » géante, chérie de tous (-tes) ${ }^{11}$, peut être ressenti comme une gêne, plus ou moins éphémère, allant à l'encontre de l'appréciation des paysages, tant pour les hommes que les femmes. En effet, les séjours en Kabylie et à Alger me permettent de dire que si "Maman, c'est sacré » (Nicette) et que « le complexe d'Ædipe est fort " (Arezki), l'idée selon laquelle l'Algérie n'aurait pas dépassé le schéma traditionnel de «domination masculine $»^{12}$ où la femme n'existerait jamais de manière autonome, son individualité étant refoulée au profit de son lien de parenté ou de son engagement matrimonial, est pour moi une représentation qui interdit de voir la réalité au risque d'assurer le silence de la pensée.

Des paysages et des habitants hantés par l'infini

La place Gueydon et le front de mer sont des lieux d'où on peut admirer le golfe de Béjaïa. Ce que j'y ai vu et entendu m'a permis de prendre conscience qu'un paysage « fini » était hanté par l'infini.

17 La place Gueydon, un balcon en ville, donne sur le port et la baie. À gauche il y a une banque, à droite la terrasse d'un café à l'européenne, en bas le port, comme si on était perché sur un arbre pour mieux admirer le spectacle que représente son activité, en face le golfe de Béjaïa, les chaînes de montagnes de la Petite Kabylie, l'horizon et le vide. Le matin, on passe y prendre un expresso, on jette rapidement un coup d'œil au loin. Dans la journée, on vient y siroter une limonade face à la mer, en famille, entre copains, entre amies, peu importe. En fin de journée, je l'ai vue pleine d'hommes : audelà d'une certaine heure (la sortie des bureaux) la femme n'a plus vraiment sa place en ville : « elle doit être chez elle, pour retrouver les enfants, etc. » (Malek). Le soir, si on y va déguster des sorbets au citron, c'est en famille ou sous couvert d'un homme. 


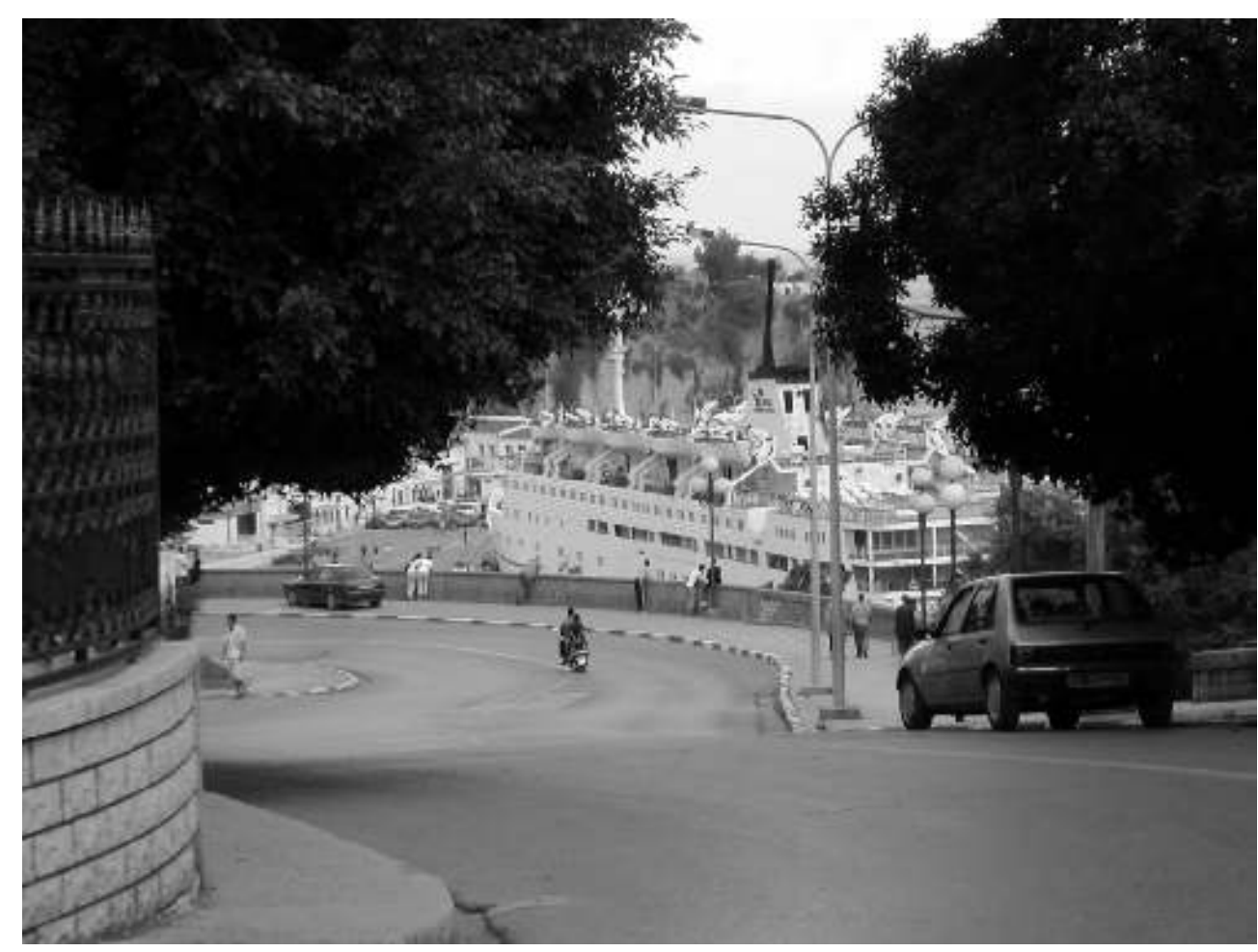

(C) Photos berki o\&m, 2002

Les dimensions de la place, sa conception, l'ouverture qu'elle crée dans cette petite ville méditerranéenne, aux vieilles rues étroites et longues, accrochée à flan de montagne, et le paysage époustouflant qu'elle permet de découvrir, lui donnent un caractère intime et immense. On est à la mer en étant en ville. On lui tourne le dos un bref instant, comme pour oublier qu'elle est prise en étau entre les montagnes kabyles, une fois de plus imprenables, la Méditerranée, limite floue ou frontière vive ouverte sur le bassin, avec d'un côté le terminal pétrolier au débouché du pipeline d'Hassi-Messaoud, de l'autre les citernes à pétrole ${ }^{13}$. C'est comme si on était poussé vers le large faute de place. La possibilité qu'on a de porter le regard au loin sans rencontrer d'obstacle et la bouffée d'air qu'on y respire réveillent les sens. La nuit on vient y admirer les lueurs dans l'obscurité. Les pétroliers en rade apparaissent à portée de main alors que le niveau de vie des habitants est sans commune mesure avec ce que pourrait leur offrir l'argent de la rente pétrolière. J'ai été fascinée par le spectacle, mais mon amertume était telle que je les ai vus comme des monstres toujours prêts à pomper sans répit l'or noir du bled qui se meurt en silence malgré les tentatives et les mobilisations à l'intérieur et hors des frontières.

19 Pour certains, regarder au loin, c'est échapper à ce qui bloquerait la vue : «Quand je regarde vers la mer, je cherche à rien voir ! Il n'y a rien qui gêne, y a pas d'horizon » (Nacer). Pour d'autres la présence du large renforce celle des montagnes: «Malgré toute la misère, les montagnes restent, la nature, le paysage est là. On a la nostalgie d'une époque paisible, sereine, presque sucrée » (Malek). Je me souviens avoir dit : «Si Béjaïa était en Europe, ce serait une ville réservée aux stars du show biz ».

20 Béjaïa la nuit, c'est aussi les lueurs des quartiers illicites dont on connaît à peine l'existence, même si on y vit toute l'année : « Il y a dix ans tout le monde se connaissait à Bougie. Ça fait vingt ans que je vis là et il $\mathrm{y}$ a des quartiers où je ne suis jamais allé » (un ami). Béjaïa une nuit d'été, c'est aussi l'invasion des jardins privés par les chats 
errants; c'est la cacophonie des chiens qui aboient, le bruit des rideaux de fer des garages, la musique qui s'échappe d'une sono parce qu'ici ou là il y a un mariage. Ce sont des hommes qui discutent assis au pied d'un lampadaire, le chant du muezzin par lequel on est réveillé à quatre heures du matin.

21 Cette note de voyage m'a aidé à réunir deux facettes en moi. J'ai compris l'aporie que je sentais entre ma personnalité et mon travail de recherche sur les représentations sociales des paysages de marais. J'ai compris que je devais reprendre l'histoire car elle n'appartient qu'à moi et que je devais en disposer en tant que telle. J'ai compris qu'il ne me restait qu'une alternative: renoncer définitivement au combat ou chercher les chemins tortueux de la liberté intérieure. Après m'être enrichie d'avoir aimé, cherché et souffert jusqu'au bout de soi, poussée par la passion (poussée vers la mort), je crois pouvoir dire qu'il est doux de vivre avec et dans la recherche conçue comme un type de liaison dynamique à une époque, celle qu'on vit.

(c) Un bateau en ville. Photo berki o\&m, 2002.

Il est vraisemblable que la plus importante découverte que j'ai faite à Béjaïa est que le paysage tel que nous l'avons hérité de la tradition géographique française est tout simplement incapable de représenter les processus réels. C'est comme si je l'avais vu fondre dans mes mains, comme si je l'avais mis au feu pour ensuite en lire les cendres où apparaissent des significations et avec lui des représentations et des images jusqu'alors inconnues.

23 J'ai compris qu'il fallait créer ses valeurs et faire évoluer la recherche, jour après jour, par un travail éthique, opiniâtre mais invisible, qui finira par produire des fruits qui donneront peut-être naissance à une nouvelle culture paysagère.

Cette note de voyage est aussi un acte de témoignage. Si j'écoutais mon cœur, je dirais : elle servira l'avenir.

\section{NOTES}

1.La Soummam est un oued de l'est algérien dont le cours passe en Kabylie avant de se jeter dans le golfe de Béjaïa. «Sa vallée encaissée est aussi un symbole. Le 20 août 1956, le Front de libération nationale (FLN) y tenait un congrès historique, à Ifri [où] les délégués définirent la stratégie de lutte armée et esquissèrent l'Algérie d'aujourd'hui. Le 20 août fait partie de la courte liste des principales commémorations nationales, avec le 5 juillet, jour de l'indépendance. [...] En 2001, des dizaines de milliers de manifestants ont empêché les [cérémonies] officielles » (Courrier International, «Rendez-nous la Révolution! », 21/8/2001).

2.Terme emprunté à Bernard Lassus.

3.Bougie ou Béjaïa, Bijaia, Vgayeth ou Begayët en kabyle.

4.Wilaya : « département » ou « province », selon les sources. L'Algérie comprend 48 wilayas, divisées en 160 daïrates (sous-préfectures) et 1541 communes. 
5.Expression que j'ai souvent entendue en août 2001 et que j'imagine être utilisée par les Kabyles par respect pour les victimes et par analogie au « printemps berbère ». 6.Jeannine Cohen : «Ceci n'est pas un carnet de terrain... Villes de Tunisie 1964-1993, deux cartes postales en quatrième dimension ", Strates $n^{\circ} 7$, p. 169.

7.François Maspero, 2001, « Deux ou trois choses que j'ai vues de l'Algérie », Le Monde interactif du 21/11/01, p. 5.

8.Expression empruntée au chanteur Djamel Allam : El hit [le mur], album Mawlud [Naissance], 1992, LPA), chanson dédiée aux hittistes, ces chômeurs qui discutent dans la rue, adossés aux murs. Selon l'Office national des statistiques (ONS) 2,3 millions d'Algériens sont au chômage (27,3\%) sur une population active estimée à 8,5 millions de personnes. 70,7 \% des demandeurs d'emploi ont moins de 30 ans.

9.F. Zorn, 1977, Mars (www.nouveau-theatre.com.fr/spectacles/mars.html), Paris, Folio-Gallimard, 1979.

10.La question a été étudiée par d'autres à propos des paysages de Toscane et de Lombardie : voir Elisabeth Crouzet-Pavan, 2001, Enfers et paradis : l'Italie de Dante et de Giotto, Albin Michel, 490 p.

11.D'après ce que les Bougiottes m'ont dit, Gouraya est un lieu de pèlerinage, de piquenique, de ballade, d'où on admire le paysage : d'un côté Béjaïa et sa baie, de l'autre la côte en direction d'Alger.

12.Voir Pierre Bourdieu, 1998, La domination masculine, Paris, Seuil, Liber, 142 p.

13.Le pétrole et le gaz naturel représentent $97 \%$ des exportations algériennes (estimation 1997, courrier international.com). L'Italie est le premier client du pays (18,8 \% des exportations) suivi par les États-Unis (14,8 \%) et par la France (11,8 \%). La jetée du port de Béjaïa, cinq kilomètres de long, a été fermée au public pendant trois ans, en raison du contexte politique instable. Plusieurs sites, dignes des " grands sites » appréciés des Bougiottes, sont aussi des sites stratégiques. Certains sont toujours réquisitionnés par l'armée, comme le phare du Cap Carbon.

\section{RÉSUMÉS}

Au-delà des clichés, que savons-nous des 31,7 millions d'Algériens (nes) qui vivent sur un territoire dont la superficie est presque quatre fois et demi celle de la France ? Difficile pour une occidentale de comprendre les paysages et la modernité dans un pays très différent du cadre dans lequel elle vit ; de voir se côtoyer l'en-dehors des normes, la volonté de tout contrôler, la rudesse et la simplicité de la vie. Beaucoup plus subtil encore est ce qui n'est pas visible pour l'œil. Si cette note de voyage peut donner le goût d'en savoir plus...

Beyond the traditional clichés, what do we know of the 31.7 million Algerians who live on a territory whose surface is almost four times and a half that of France? It has been difficult for a Westerner like me to understand the landscapes and the modernity of a country which is very different from the environment I live in. It is as difficult to see how harshness, the ab-normal, a will to control everything and a simplicity of life coexist. What is not visible for the eye is even more subtle. May this field note arouse your curiosity... 
INDEX

Mots-clés : Paysage

Index géographique : Algérie

\section{AUTEUR}

ODILE BERKI

Géographe, doctorante, Ladyss, université de Paris 1, odile.berki@libertysurf.fr 